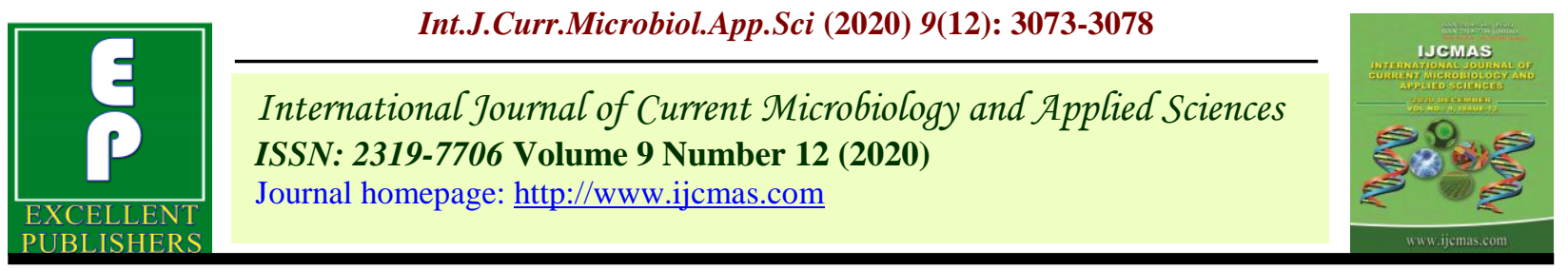

\title{
Impact of Varieties and Integrated Nutrient Management on Incidence of Aphid (Aphis gossipy Glover) on Isabgol (Plantago ovata Forsk.) under Northern Dry Zone of Karnataka
}

\author{
Siddalingayya V. Salimath*, Y. K. Kotikal, K. N. Kattimani, \\ N. S. Nagarja, Jameel Jhalegar, D. R. Patil and J. Venkatesh \\ College of Horticulture, University of Horticultural Sciences, Bagalkot, India \\ *Corresponding author
}

\section{A B S T R A C T}

Keywords

Aphis gossipy,

INM, Gujarat

Isabgol-2, Vallabh

Isabgol-1

Article Info

Accepted:

22 November 2020

Available Online:

10 December 2020

\begin{abstract}
The field experiment was conducted to evaluate the performance of two Isabgol cultivars and INM on the incidence of aphids in northern dry zone of Karnataka during 2015-16 and 2016-17. The experiment was laid out in split plot design (SPD) with sixteen INM treatment combinations at the college of horticulture, Bagalkot. Pooled data analysis of two years data exhibited lower aphid (Aphis gossypii Glover) incidence in Vallabha Isabgol-1 with integrated nutrient management. The lower aphid incidence was recorded with supply of $75 \% \mathrm{RD}$ of FYM $+75 \% \mathrm{RD}$ of $\mathrm{NPK}+$ Azospirillum $+P S B+\mathrm{ZnSO}_{4}+$ $\mathrm{FeSO}_{4}\left(\mathrm{~N}_{11}\right)$ and maximum aphid incidence was recorded with supply of $50 \% \mathrm{RD}$ of $\mathrm{FYM}+50 \% \mathrm{RD}$ of NPK + Azotobacter $\left(\mathrm{N}_{13}\right)$ at 90 DAS. In interaction effect minimum aphid infestation was recorded in Vallabh Isabgol-1, supplied with Vermicompost $+50 \%$ $\mathrm{RD}$ of NPK + Azospirillum + Azotobacter $\left(\mathrm{V}_{1} \mathrm{~N}_{6}\right)$ followed by Vallabh Isabgol-1, $75 \%$ $\mathrm{RD}$ of $\mathrm{FYM}+75 \% \mathrm{RD}$ of $\mathrm{NPK}+$ Azospirillum $+P S B+\mathrm{ZnSO}_{4}+\mathrm{FeSO}_{4}\left(\mathrm{~V}_{1} \mathrm{~N}_{11}\right)$ as compared to other treatment. The maximum aphid incidence was recorded in Gujarat Isabgol-2, supplied with RD of FYM + RD of NPK $\left(\mathrm{V}_{2} \mathrm{~N}_{1}\right)$.
\end{abstract}

\section{Introduction}

Isabgol seeds and husk are mainly used in medicine because of mucilaginous nature. It is used as safe bulk-laxative, promoting regular bowel movement. It is a popular medicinal crop in Ayurvedic and Unani systems of medicine for problems like chronic diarrhoea, dysentery, constipation, reducing cholesterol and treatment of cancer. Recent studies have shown that, an intake of $10.2 \mathrm{~g}$ of husk per day as part of a diet significantly lowers the blood cholesterol level. Since 1998, the US Food and Drug Administration approved the health claim on the association between consumption of Isabgol husk and reduction in risk of coronary heart disease. The seeds are also used for 2 the treatment of chronic amoebic dysentery, where constipation is one of the main symptoms (Kumar et al., 2015).

India exports 400 corers Isabgol annually. As the whole, India holds monopoly in the production and export of isabgol to the world 
market and about 80 to 90 per cent produce is mainly exported to U.S.A., West Germany, United Kingdom and France. Isabgol is commercially grown as a winter crop, which remain in the field for about four to five months.

The most important component of isabgol is husk obtained from its seed. Isabgol husk is popularly known as "Sat isabgol" in Indian market. It is an important medicine for intestinal and stomach disorders. Isabgol crop is attacked by number of insect pest, out of which aphid, Aphis gossypii Glover (Homoptera: Aphididae) has been reported as major pest of isabgol (Sagar and Jindla, 1984).

A. gossypii is a polyphagous pest which infesting number of field crops. The work on A. gossypii was carried out by various workers on different hosts in India as well as in abroad. A. gossypii, which has been observed to attack 220 host plant belonging to 46 families throughout the world (Roy and Behura, 1983). Present practices followed for the management of aphids was use of synthetic pesticides.

The continuous and indiscriminate use of synthetic pesticides cause the problem of resistance, resurgence, environmental pollution, health hazards, harmful residues on food crop and adverse effect on activity of parasites and predator of crop pests.

Inspite of regular occurrence of $A$. gossypii on isabgol crop in North Gujarat and South Rajasthan regions causing economic losses, no any systemic work has been done on various aspects of such an important pest of isabgol in Gujarat State. Keeping this in view, to overcome lacunae and to develop an ecofriendly and effective pest management strategy, the present study was undertaken.

\section{Materials and Methods}

The experiment was conducted in the field at department of plantation spices medicinal and aromatic plants, college of horticulture, Bagalkot at Haveli farm during the years 2015-16 and 2016-17. Geographically, this experimental site lies in northern dry zone (Zone-3) of Karnataka state within the agroclimatic zone of Karnataka, situated at $16^{\circ}$ North latitude and 74'59' East longitude and at an altitude of $533.0 \mathrm{~m}$ above mean sea level. The soil of experimental field was red clay loamy in texture, with percentages of sand 22.60 , silt 26.10 and clay52.20 bulk density 1.25 , EC $0.24 \mathrm{dS} \mathrm{m}^{-1}$ and $\mathrm{pH} 8.22$ (alkaline in reaction) with organic carbon 1.63 and available 268.02, 34.80, 273.69 NPK kg $\mathrm{ha}^{-1}$. The source of seed collection was DMAPR Anandh Gujarat i.e. Vallabh Isabgol-1( $\left(\mathrm{V}_{1}\right)$ and Gujarat Isabgol-2 $\left(\mathrm{V}_{2}\right)$. Seed were sown in 18 November 2015 and 2016 with gross plot size of $3.6 \mathrm{~m} \times 1.5 \mathrm{~m}=$ $5.40 \mathrm{~m}^{2}$ in split plot design in sixteen INM sub treatments with three replications as subplot listed below.

$\mathbf{N}_{1}$-RDF FYM (10 $\left.\mathrm{t} \mathrm{ha}^{-1}\right)+$ RDF NPK (50:25:30 $\left.\mathrm{kg} \mathrm{ha}^{-1}\right)$,

$\mathbf{N}_{2}$-RDF FYM (0 $\left.\mathrm{t} \mathrm{ha}^{-1}\right)+$ RDF NPK (50:25:30 kg ha $\left.{ }^{-1}\right)+\mathrm{ZnSo}_{4}\left(15 \mathrm{~kg} \mathrm{ha}^{-1}\right)$,

$\mathbf{N}_{3}$-RDF FYM (10 $\left.\mathrm{t} \mathrm{ha}^{-1}\right)+$ RDF NPK $\left(50: 25: 30 \mathrm{~kg} \mathrm{ha}^{-1}\right)+\mathrm{FeSo}_{4}\left(7.5 \mathrm{~kg} \mathrm{ha}^{-1}\right)$,

$\mathbf{N}_{4}$-RDF FYM (10 $\left.\mathrm{t} \mathrm{ha}^{-1}\right)+$ RDF NPK $\left(50: 25: 30 \mathrm{~kg} \mathrm{ha}^{-1}\right)+\mathrm{FeSo}_{4}\left(7.5 \mathrm{~kg} \mathrm{ha}^{-1}\right)+$ $\mathrm{ZnSo}_{4}\left(15 \mathrm{~kg} \mathrm{ha}^{-1}\right)$,

$\mathbf{N}_{5}$-Vermicompost $\left(1 \mathrm{t} \mathrm{ha}{ }^{-1}\right)+$ RDF NPK $\left(50: 25: 30 \mathrm{~kg} \mathrm{ha}^{-1}\right)$

$\mathbf{N}_{6}$-Vermicompost $\left(1 \mathrm{t} \mathrm{ha}^{-1}\right)+50 \%$ RDF NPK $\left(50: 25: 30 \mathrm{~kg} \mathrm{ha}^{-1}\right)+$ Azospirillum $\left(5 \mathrm{~kg} \mathrm{ha}^{-1}\right)+$ Azotobacter $\left(5 \mathrm{~kg} \mathrm{ha}^{-1}\right)$, 
$\mathrm{N}_{7}-75 \%$ RDF FYM $\left(7.5 \mathrm{t} \mathrm{ha}^{-1}\right)+75 \%$ RDF NPK $\left(37.5: 18.75: 22.50 \mathrm{~kg} \mathrm{ha}^{-1}\right), \quad \mathrm{N}_{8}-75 \%$ RDF FYM (7.5 $\left.\mathrm{t} \mathrm{ha}^{-1}\right)+75 \%$ RDF NPK (37.5:18.75:22.50 kg ha $\left.{ }^{-1}\right)+$ Azotobacter $(5 \mathrm{~kg}$ $\left.\mathrm{ha}^{-1}\right)$,

$\mathrm{N}_{9}-75 \%$ RDF FYM $\left(7.5 \mathrm{t} \mathrm{ha}^{-1}\right)+75 \%$ RDF NPK $\quad\left(37.5: 18.75: 22.50 \quad \mathrm{~kg} \quad \mathrm{ha}^{-1}\right)+$ Azospirillum $\left(5 \mathrm{~kg} \mathrm{ha}^{-1}\right)$,

$\mathrm{N}_{10}-75 \%$ RDF FYM $\left(7.5 \mathrm{t} \mathrm{ha}^{-1}\right)+75 \%$ RDF NPK $\left(37.5: 18.75: 22.50 \mathrm{~kg} \mathrm{ha}^{-1}\right)+P S B(3 \mathrm{~kg}$ $\left.\mathrm{ha}^{-1}\right), \mathrm{N}_{11}-75 \%$ RDF FYM $\left(7.5\right.$ tha $\left.^{-1}\right)+75 \%$ RDF NPK (37.5:18.75:22.50 kg ha $\left.{ }^{-1}\right)+$ Azospirillum $\left(5 \mathrm{~kg} \quad \mathrm{ha}^{-1}\right)+\quad P S B \quad(3 \mathrm{~kg}$ $\left.\mathrm{ha}^{-1}\right)+\mathrm{ZnSO}_{4}\left(15 \mathrm{~kg} \mathrm{ha}^{-1}\right)+\mathrm{FeSO}_{4}(7.5 \mathrm{~kg}$ $\left.\mathrm{ha}^{-1}\right) \mathrm{N}_{12}-50 \%$ RDF FYM $\left(5 \mathrm{tha}^{-1}\right)+50 \%$ RDF NPK (25:12.5:15 NPK kg ha $\left.{ }^{-1}\right)$

$\mathrm{N}_{13}-50 \%$ RD FYM $\left(5 \mathrm{tha}^{-1}\right)+50 \%$ RDF NPK $\left(25: 12.5: 15 \mathrm{~kg} \mathrm{ha}^{-1}\right)+$ Azotobacter $\left(5 \mathrm{~kg} \mathrm{ha}^{-1}\right)$,

$\mathrm{N}_{14}-50 \%$ RDF FYM $\left(5 \mathrm{tha}^{-1}\right)+50 \%$ RDF NPK (25:12.5:15 kg ha-1 $)+$ Azospirillum $(5 \mathrm{~kg}$ $\left.\mathrm{ha}^{-1}\right), \mathrm{N}_{15}-50 \%$ RDF FYM $\left(5 \mathrm{t} \mathrm{ha}^{-1}\right)+50 \%$ RDF NPK $\left(25: 12.5: 15 \quad \mathrm{~kg} \quad \mathrm{ha}^{-1}\right) \quad+P S B$ $\left(3 \mathrm{~kg} \mathrm{ha}^{-1}\right)$,

$\mathrm{N}_{16}-50 \%$ RD FYM $\left(5 \mathrm{tha}^{-1}\right)+75 \%$ RDF NPK $\left(37.5: 18.75: 22.50 \mathrm{~kg} \mathrm{ha}^{-1}\right)+$ Azospirillum $\left(5 \mathrm{~kg} \mathrm{ha}^{-1}\right)+P S B\left(3 \mathrm{~kg} \mathrm{ha}^{-1}\right)+\mathrm{Znso}_{4}$

$\left(15 \mathrm{~kg} \mathrm{ha}^{-1}\right)+\mathrm{FeSO}_{4}\left(7.5 \mathrm{~kg} \mathrm{ha}^{-1}\right)$.

Azotobacter (Azotobacter sp. $02 \quad$ X10-9 $\mathrm{cfu} / \mathrm{g}$ ), Azospirillum (Azospirillum sp., 02 X10-9 cfu/g) and Phosphate solubilising bacteria (Bacillus megaterium $02 \quad$ X10-9 cfu/g) produced in the UHS Bagalkot biocontrol laboratory. The experiment after layout then treatments were applied as per above nutrient combination, then mixed thoroughly in plots before imposing the treatments. Zinc was applied in the form of $\mathrm{ZnSO}_{4}$ at the time of sowing half dose of $\mathrm{N}$ was applied as a basal and remaining half was applied one month after sowing as top dressing full dose of $\mathrm{P}$ and $\mathrm{K}$ were applied at the time of sowing below the seed in furrows made with the help of land hoe. Manual thinning weeding and hoeing were done at one month after sowing to provide an ideal environment to the crop a light irrigation was given immediately before sowing, however six and seven irrigation were given as pet requirement of the crop with the help of sprinkler as per package of practices cropping pattern and its management have been taken Scoring of aphid infection was done at 30,60 and 90 days after planting in two years by following aphid index given by the Bank (1954) were fixed for estimating the population of aphid and the average aphid index was worked out by adopting following formula.

Average aphid Index plant ${ }^{-1}=$

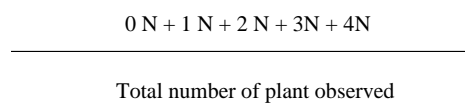

Where, 0, 1, 2, 3, 4 are aphid index

$\mathrm{N}=$ Number of plant showing respective aphid index

Aphid Index
0
1
2
3
4

Degree of infestation

Plant free from aphid

Aphid present, but colonies did not build up. No injury due to pest apparent on the plant Small colonies of aphid were present

Large colonies of aphid were present on tender parts. Counts of aphids in colonies were possible and tender plant part show damage symptom due to aphids

Entire plant was covered by aphids. Counts of aphids in colonies were impossible and plant show damage symptom due to aphids 


\section{Results and Discussion}

Aphid (Aphis gossypii Glover) infestation was calculated with score of $0-4$. The least aphid incidence was recorded in Vallabh Isabagol-1 (2.28, 2.2.39 and 2.34) and highest aphid incidence was recorded in Gujarat Isabgol-2 (2.41, 2.51 and 2.46) during 2015-16, 201617 and pooled data respectively.

The highest aphid incidence was recorded in Gujarat Isabgol-2 (2.41) during 2015-16, 2016-17 and pooled data at all the stages of crop growth.

At 30 DAS, aphid infestation was recorded but colonies did not build up to cause any injury to crops due to pest apparent on the plant.

But during other stages of crop growth viz. 60 and 90 DAS it was found that aphids were present in small colonies this result minimum incidence and varietal response. Similar findings were recorded by Sagar et al., (1987), Prajapati and Yadav (2005).

The aphid incidence was influenced by integrated nutrient management at 90 DAS. Significantly minimum incidence $(1.55,1.67)$ was recorded with application of $\mathrm{N}_{11}(75 \%$ $\mathrm{RD}$ of $\mathrm{FYM}+75 \% \mathrm{RD}$ of $\mathrm{NPK}+$ Azospirillum $\left.+P S B+\mathrm{ZnSO}_{4}+\mathrm{FeSO}_{4}\right)$, which was on par with $\mathrm{N}_{6}(1.6,1.70), \mathrm{N}_{4}$ $(1.67,1.77$.$) and \mathrm{N}_{16}(1.73,1.86)$.

Maximum aphid infestation was $(2.77,2.85)$ recorded with application of $\mathrm{N}_{13}(50 \% \mathrm{RD}$ of $\mathrm{FYM}+50 \%$ RD of NPK + Azatobacter) during 2015, 2016 respectively.

Similar trend was recorded in pooled data. INM helps release of nutrients at faster rate results plants healthy growth and showing minimum resistance to insects. Similar findings reported by Patel et al., 2014.
The aphid infestation was influenced by varieties and integrated nutrient management at 90 DAS. Significantly less aphid incidence $(1.45,1.55)$ was recorded in Vallabh Isabgol1 supplied with $\mathrm{N}_{6}$ (Vermicompost $+50 \%$ RD of NPK + Azospirillum + Azatobacter), which were on par with $\mathrm{V}_{2} \mathrm{~N}_{16}(1.52,1.62)$, $\mathrm{V}_{1} \mathrm{~N}_{11}(1.54,2.59)$ and $\mathrm{V}_{2} \mathrm{~N}_{11}(1.56,1.66)$.

Higher aphid incidence $(2.86,2.78)$ was recorded in $\left(\mathrm{V}_{1} \mathrm{~N}_{11}\right)$ Vallabh Isabgol-1, supplied with $50 \%$ RD of FYM + 50\% RD of NPK + Azatobacter during 2015, 2016 respectively. Similar trend was recorded in pooled data.

The aphid infestation influenced by varieties, integrated nutrient management at 90 DAS. Significantly less aphid incidence was recorded in Vallabh Isabgol-1 supplied with $\mathrm{T}_{6}$ (Vermicompost $+50 \%$ RD NPK + ASP + AZB) (1.45), which were on par with $\mathrm{V}_{2} \mathrm{~T}_{16}$ (1.52), $\mathrm{V}_{1} \mathrm{~T}_{11}$ (1.54) and $\mathrm{V}_{2} \mathrm{~T}_{11}$ (1.56).

More aphid incidence was recorded in Vallabh Isabgol-1 $\left(\mathrm{T}_{13)}\right.$ supplied with 50\% RD $\mathrm{FYM}+50 \%$ RD NPK + AZB (2.86) during 2015-16.

Similar trend was recorded 2016-17, pooled data (Table-1 and Figure-1).

The varieties and integrated nutrient management significantly influenced by aphid infestation at 90DAS. Significantly less aphid infestation was recorded in Vallabh Isabgol-1, supplied with Vermicompost $+50 \% \mathrm{RD}$ of $\mathrm{NPK}+$ Azospirillum + Azotobacter $\left(\mathrm{V}_{1} \mathrm{~N}_{6}\right)$ followed by Vallabh Isabgol-1, $75 \%$ RD of $\mathrm{FYM}+75 \% \mathrm{RD}$ of NPK + Azospirillum + $P S B+\mathrm{ZnSO}_{4}+\mathrm{FeSO}_{4}\left(\mathrm{~V}_{2} \mathrm{~N}_{11}\right)$ as compared to other treatment combinations. 
Table.1 Aphids infestation as influenced by Isabgol varieties and integrated nutrient management (0-4 Scale)

\begin{tabular}{|c|c|c|c|c|c|c|c|c|c|c|c|c|c|c|c|c|c|c|c|c|c|c|c|c|c|c|c|}
\hline \multirow{3}{*}{$\begin{array}{c}6 \\
\text { Nutrients }\end{array}$} & \multicolumn{9}{|c|}{2015} & \multicolumn{9}{|c|}{2016} & \multicolumn{9}{|c|}{ Pooled data } \\
\hline & \multicolumn{3}{|c|}{30 DAS } & \multicolumn{3}{|c|}{60 DAS } & \multicolumn{3}{|c|}{90 DAS } & \multicolumn{3}{|c|}{30 DAS } & \multicolumn{3}{|c|}{60 DAS } & \multicolumn{3}{|c|}{90 DAS } & \multicolumn{2}{|c|}{30 DAS } & \multicolumn{3}{|c|}{60 DAS } & \multicolumn{4}{|c|}{90 DAS } \\
\hline & $V_{1}$ & $\mathbf{v}_{2}$ & Mean & $V_{1}$ & $\mathbf{v}_{2}$ & Mean & $\mathrm{V}_{1}$ & $v_{2}$ & Mean & $\mathrm{V}_{1}$ & $\mathbf{v}_{2}$ & Mean & $\mathrm{V}_{1}$ & $\mathrm{v}_{2}$ & Mean & $\mathrm{v}_{1}$ & $\mathbf{v}_{2}$ & $\begin{array}{c}\text { Mea } \\
\mathbf{n}\end{array}$ & $v_{1}$ & $\mathbf{V}_{2}$ & Mean & $\mathbf{V}_{1}$ & $V_{2}$ & Mean & $\mathbf{V}_{1}$ & $V_{2}$ & Mean \\
\hline$N_{1}$ & 1.36 & 1.41 & 1.38 & 1.95 & 2.09 & 2.02 & 2.48 & 2.78 & 2.63 & 1.40 & 1.57 & 1.49 & 1.97 & 2.16 & 2.07 & 2.59 & 2.97 & 2.78 & 1.38 & 1.49 & 1.43 & 1.96 & 2.13 & 2.04 & 2.53 & 2.87 & 2.70 \\
\hline $\mathbf{N}_{2}$ & 1.40 & 1.57 & 1.48 & 1.96 & 1.89 & 1.92 & 2.45 & 2.44 & 2.44 & 1.56 & 1.41 & 1.48 & 2.06 & 2.02 & 2.04 & 2.53 & 2.54 & 2.53 & 1.48 & 1.49 & 1.48 & 2.01 & 1.95 & 1.98 & 2.49 & 2.49 & 2.49 \\
\hline $\mathrm{N}_{3}$ & 1.28 & 1.61 & 1.45 & 1.77 & 2.11 & 1.94 & 2.30 & 2.68 & 2.49 & 1.31 & 1.68 & 1.50 & 1.91 & 2.28 & 2.09 & 2.38 & 2.78 & 2.58 & 1.29 & 1.65 & 1.47 & 1.84 & 2.20 & 2.02 & 2.34 & 2.73 & 2.54 \\
\hline $\mathbf{N}_{4}$ & 0.90 & 0.82 & 0.86 & 1.25 & 1.28 & 1.26 & 1.60 & 1.73 & 1.67 & 0.95 & 0.84 & 0.89 & 1.32 & 1.38 & 1.35 & 1.70 & 1.83 & 1.77 & 0.93 & 0.83 & 0.88 & 1.28 & 1.33 & 1.30 & 1.65 & 1.78 & 1.72 \\
\hline $\mathbf{N}_{5}$ & 1.47 & 0.84 & 1.15 & 2.06 & 1.75 & 1.91 & 2.46 & 2.45 & 2.46 & 1.74 & 1.26 & 1.50 & 2.23 & 1.86 & 2.05 & 2.56 & 2.55 & 2.56 & 1.61 & 1.05 & 1.33 & 2.15 & 1.81 & 1.98 & 2.51 & 2.50 & 2.51 \\
\hline $\mathbf{N}_{6}$ & 0.91 & 0.62 & 0.76 & 1.18 & 1.19 & 1.18 & 1.45 & 1.76 & 1.60 & 0.92 & 0.64 & 0.78 & 1.45 & 1.26 & 1.36 & 1.55 & 1.86 & 1.70 & 0.91 & 0.63 & 0.77 & 1.31 & 1.23 & 1.27 & 1.50 & 1.81 & 1.65 \\
\hline $\mathbf{N}_{7}$ & 1.44 & 1.70 & 1.57 & 1.95 & 2.11 & 2.03 & 2.43 & 2.66 & 2.55 & 1.52 & 1.64 & 1.58 & 2.28 & 2.28 & 2.28 & 2.57 & 2.73 & 2.65 & 1.48 & 1.67 & 1.57 & 2.12 & 2.20 & 2.16 & 2.50 & 2.70 & 2.60 \\
\hline $\mathrm{N}_{8}$ & 1.45 & 1.69 & 1.57 & 1.91 & 2.13 & 2.02 & 2.38 & 2.67 & 2.52 & 1.49 & 1.69 & 1.59 & 2.41 & 2.26 & 2.34 & 2.48 & 2.77 & 2.62 & 1.47 & 1.69 & 1.58 & 2.16 & 2.20 & 2.18 & 2.43 & 2.72 & 2.57 \\
\hline $\mathrm{N}_{9}$ & 1.67 & 1.59 & 1.63 & 2.18 & 1.97 & 2.08 & 2.69 & 2.52 & 2.61 & 1.73 & 1.62 & 1.68 & 2.25 & 2.07 & 2.16 & 2.77 & 2.62 & 2.70 & 1.70 & 1.61 & 1.65 & 2.21 & 2.02 & 2.12 & 2.73 & 2.57 & 2.65 \\
\hline $\mathrm{N}_{10}$ & 1.61 & 1.68 & 1.64 & 2.20 & 2.19 & 2.20 & 2.65 & 2.74 & 2.70 & 1.82 & 1.84 & 1.83 & 2.36 & 2.33 & 2.35 & 2.77 & 2.91 & 2.84 & 1.71 & 1.76 & 1.74 & 2.28 & 2.26 & 2.27 & 2.71 & 2.83 & 2.77 \\
\hline $\mathrm{N}_{11}$ & 0.85 & 0.85 & 0.85 & 1.19 & 1.21 & 1.20 & 1.54 & 1.56 & 1.55 & 0.86 & 0.95 & 0.90 & 1.29 & 1.34 & 1.32 & 1.67 & 1.66 & 1.67 & 0.85 & 0.90 & 0.88 & 1.24 & 1.27 & 1.26 & 1.60 & 1.61 & 1.61 \\
\hline$N_{12}$ & 1.71 & 1.88 & 1.79 & 2.55 & 2.29 & 2.42 & 2.65 & 2.64 & 2.65 & 1.86 & 2.45 & 2.15 & 2.65 & 2.39 & 2.52 & 2.74 & 2.74 & 2.74 & 1.79 & 2.16 & 1.97 & 2.60 & 2.34 & 2.47 & 2.69 & 2.69 & 2.69 \\
\hline$N_{13}$ & 1.92 & 1.92 & 1.92 & 2.62 & 2.78 & 2.70 & 2.68 & 2.86 & 2.77 & 1.96 & 1.97 & 1.96 & 2.72 & 2.88 & 2.80 & 2.78 & 2.93 & 2.85 & 1.94 & 1.95 & 1.94 & 2.67 & 2.83 & 2.75 & 2.73 & 2.90 & 2.81 \\
\hline $\mathrm{N}_{14}$ & 1.58 & 1.95 & 1.77 & 2.62 & 2.67 & 2.64 & 2.55 & 2.70 & 2.62 & 1.94 & 1.88 & 1.91 & 2.81 & 2.77 & 2.79 & 2.65 & 2.80 & 2.72 & 1.76 & 1.92 & 1.84 & 2.71 & 2.72 & 2.71 & 2.60 & 2.75 & 2.67 \\
\hline $\mathbf{N}_{15}$ & 1.95 & 1.95 & 1.95 & 2.14 & 2.85 & 2.50 & 2.32 & 2.77 & 2.55 & 1.96 & 1.96 & 1.96 & 2.37 & 2.94 & 2.66 & 2.42 & 2.91 & 2.66 & 1.96 & 1.96 & 1.96 & 2.26 & 2.90 & 2.58 & 2.37 & 2.84 & 2.61 \\
\hline$N_{16}$ & 0.86 & 0.79 & 0.83 & 1.40 & 1.16 & 1.28 & 1.93 & 1.52 & 1.73 & 0.96 & 0.84 & 0.90 & 1.56 & 1.20 & 1.38 & 2.09 & 1.62 & 1.86 & 0.91 & 0.82 & 0.87 & 1.48 & 1.18 & 1.33 & 2.01 & 1.57 & 1.79 \\
\hline \multirow[t]{2}{*}{ MEAN } & 1.40 & 1.43 & & 1.93 & 1.98 & & 2.28 & 2.41 & & 1.50 & 1.52 & & 2.10 & 2.09 & & 2.39 & 2.51 & & 1.45 & 1.47 & & 2.02 & 2.03 & & 2.34 & 2.46 & \\
\hline & $\begin{array}{l}\text { S.Em } \\
\pm\end{array}$ & \multicolumn{2}{|c|}{ C.D at $5 \%$} & S.Em \pm & \multicolumn{2}{|c|}{ C.D at $5 \%$} & $\begin{array}{l}\text { S.Em } \\
\pm\end{array}$ & \multicolumn{2}{|c|}{ C.D at $5 \%$} & S.Em \pm & \multicolumn{2}{|c|}{ C.D at $5 \%$} & $\begin{array}{l}\text { S.Em } \\
\quad \pm\end{array}$ & \multicolumn{2}{|c|}{ C.D at $5 \%$} & $\begin{array}{l}\text { S.Em } \\
\pm\end{array}$ & \multicolumn{2}{|c|}{ C.D at $5 \%$} & S.Em \pm & $\begin{array}{l}\text { C.D at } \\
5 \%\end{array}$ & $\begin{array}{l}\text { S.E } \\
\mathrm{m} \pm\end{array}$ & \multicolumn{2}{|c|}{ C.D at $5 \%$} & $\begin{array}{l}\text { S.Em } \\
\pm\end{array}$ & \multicolumn{3}{|c|}{ C.D at $5 \%$} \\
\hline Varieties (V) & 0.007 & \multicolumn{2}{|c|}{ NS } & 0.05 & & IS & 0.07 & & & 0.002 & & & 0.056 & & vS & 0.07 & 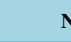 & & 0.003 & 0.009 & 0.05 & & NS & 0.07 & & NS & \\
\hline $\begin{array}{l}\text { Nutrients } \\
\text { (N) }\end{array}$ & 0.06 & & & 0.05 & & 15 & 0.07 & & & 0.07 & & & 0.064 & & 18 & 0.07 & 0. & & 0.05 & 0.16 & 0.05 & & 0.15 & 0.07 & & 0.20 & \\
\hline $\mathrm{N}$ at same $\mathrm{V}$ & 0.08 & & & 0.07 & & 21 & 0.10 & & & 0.10 & & & 0.090 & & .26 & 0.09 & 0. & & 0.07 & 0.22 & 0.07 & & 0.22 & 0.09 & & 0.28 & \\
\hline $\begin{array}{l}\text { Vat same or } \\
\text { different } N\end{array}$ & 0.03 & & & 0.20 & & 57 & 0.28 & & & 0.02 & & & 0.219 & & .62 & 0.30 & 0. & & 0.02 & 0.07 & 0.21 & & 0.59 & 0.29 & & 0.83 & \\
\hline
\end{tabular}


Fig.1 Aphids infestation as influenced by Isabgol varieties and integrated nutrient management

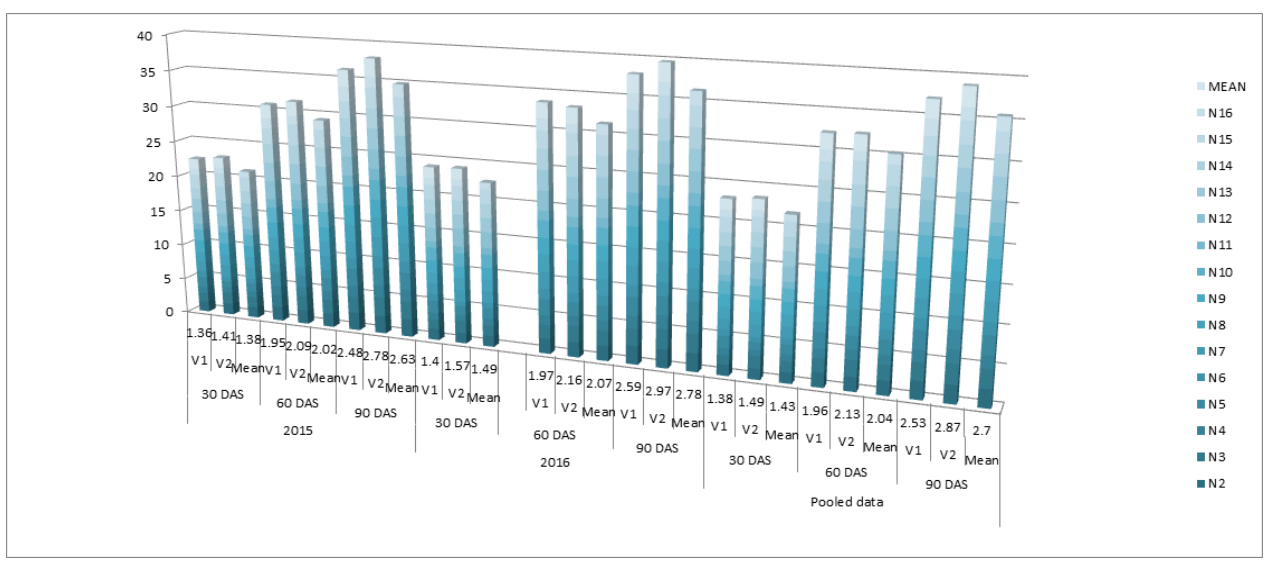

The maximum aphid incidence was recorded in Gujarat Isabgol-2, supplied with RD of $\mathrm{FYM}+\mathrm{RD}$ of NPK $\left(\mathrm{V}_{2} \mathrm{~N}_{1}\right)$. The higher aphid infestation was recorded in Gujarat Isabgol-2, supplied with RD of FYM + RD of NPK $\left(\mathrm{V}_{2} \mathrm{~N}_{1}\right)$ due to varietal characters and more nutrients absorption helps to build resistance mechanism towards pest.

\section{References}

Annual Report. 2019, DMAPR.

Farooqui, A. A. and Sreeramu, B. S., 2001, A text book an cultivation of medicinal and aromatic crops. Universities Press (India) Ltd., Hyderabad. Pp. 168-174.

Sagar, P., Jindla, L. N. and Mehta, S. K., 1987, Field screening of Isabgol
(Plantago ovata Linn.) against aphid, (Aphis gossypii Glover) in Punjab. J. Res. PAU., 24 (3): 441-444.

Patel, S. R., Patel M. V. and Parmar. V. R., 2014, Influence of plant height and width on population of aphid, (Aphis gossypii Glover) infesting Isabgol. Trends in Bioscience., 7(24): 4494.

Prajapati, K. R. and Yadav, H. S., 2005, Screening of Isabgol varieties against Aphid spp. Annual Report, p. 10-11.

Roy, D. K. and Behura, B. K. 1983. Notes on host plants, feeding behavior, infestation and ant attendance of cotton aphids, Aphis gossypii Glover. J. Bombay Natural Hist. Soc. 80(3): 654656.

\section{How to cite this article:}

Siddalingayya V. Salimath, Y. K. Kotikal, K. N. Kattimani, N. S. Nagarja, Jameel Jhalegar, D. R. Patil and Venkatesh, J. 2020. Impact of Varieties and Integrated Nutrient Management on Incidence of Aphid (Aphis gossipy Glover) on Isabgol (Plantago ovata Forsk.) under Northern Dry Zone of Karnataka. Int.J.Curr.Microbiol.App.Sci. 9(12): 3073-3078. doi: https://doi.org/10.20546/ijcmas.2020.912.364 